Proyecciones Journal of Mathematics

Vol. 33, $\mathrm{N}^{\circ}$ 4, pp. 471-482, December 2014.

Universidad Católica del Norte

Antofagasta - Chile

\title{
Topological indices of Kragujevac trees
}

\author{
Roberto Cruz \\ Universidad de Antioquia, Colombia \\ Iván Gutman \\ University of Kragujevac, Serbia \\ and \\ Juan Rada \\ Universidad de Antioquia, Colombia \\ Received : May 2014. Accepted : November 2014
}

\begin{abstract}
We find the extremal values of the energy, the Wiener index and several vertex-degree-based topological indices over the set of Kragujevac trees with the central vertex of fixed degree.
\end{abstract}

2010 Mathematics Subject Classification : 05C90, $05 C 35$.

Key Words : Topological indices; Kragujevac trees. 


\section{Introduction}

The class of Kragujevac trees emerged in several studies addressed to solve the problem of characterizing the tree with minimal atom-bond connectivity index $[6,11,12]$. In this work, we are interested in finding the maximal and minimal value of several topological indices over a subset of Kragujevac trees of order $n$.

Let $G$ be a simple graph on $n$ vertices. By $u v$ we denote the edge connecting the vertices $u$ and $v$. A vertex of degree one is referred to as a pendent vertex. An edge whose one end-vertex is pendent is referred to as a pendent edge.

The formal definition of a Kragujevac tree was introduced in [15]:

Let $B_{1}, B_{2}, B_{3}, \ldots$ be branches whose structure is depicted in Figure 1 . A proper Kragujevac tree is a tree possessing a central vertex of degree at least 3 , to which branches of the form $B_{1}$ and/or $B_{2}$ and/or $B_{3}$ and/or ... are attached. The set of all proper Kragujevac trees of order $n$ will be denoted by $\mathbf{K g}_{n}$. An improper Kragujevac tree is a tree obtained by inserting a new vertex (of degree 2) on a pendent edge of a proper Kragujevac tree. The set of all improper Kragujevac trees of order $n$ will be denoted by $\mathbf{K g}_{n}^{*}$.

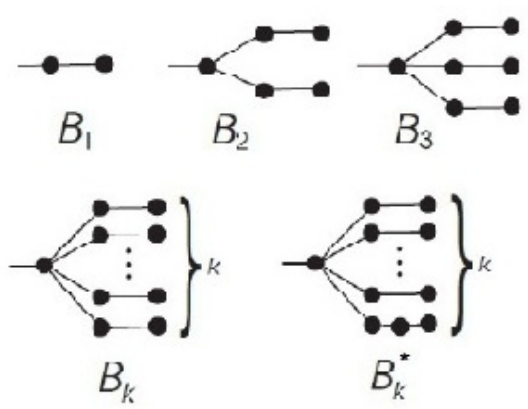

\section{Figure 1 : The branches of Kragujevac trees}

Let $\tilde{\mathbf{K}} \mathbf{g}_{n, m}$ be the class of proper Kragujevac trees of order $n$ with the central vertex of degree $m$, and no branches of type $B_{1}$. In this case, the values of $n$ and $m$ have different parity and are related by the formula $\mathrm{n}=\mathrm{m}+1+2 \sum_{i=1}^{m} k_{i}$, where $3 \leq m \leq \frac{n-1}{5}$. The branches attached to the central vertex are of the form $B_{k_{1}}, B_{k_{2}}, \ldots B_{k_{m}}$ where $k_{i} \geq 2$ for all $i=$ 
$1, \ldots, m$. An interesting property of this class is the fact that from any specific tree one can obtain any other tree of the family by moving pendent edges from one branch to another.

Given two branches $B_{k_{i}}$ and $B_{k_{j}}$, the gap between them is defined as $\left|k_{i}-k_{j}\right|$ and the gap of a tree $T \in \tilde{\mathbf{K}} \mathbf{g}_{n, m}$ is defined as $g(T)=\max _{1 \leq i<j \leq m}\left|k_{i}-k_{j}\right|$.

The tree with maximal gap is the tree, denoted by $U$, with $m-1$ branches of type $B_{2}$ and one branch of type $B_{h}$, where $h=\frac{n+3-5 m}{2}$


Figure 2 : Trees of $\tilde{\mathbf{K}} g_{n, m}$ with maximal and minimal gaps

On the other hand, the tree with minimal gap is the tree, denoted by $V$, in which all the branches $B_{k_{1}}, B_{k_{2}}, \ldots, B_{k_{m}}$ satisfy the condition $\left|k_{i}-k_{j}\right| \leq 1$. In this case $g(V) \leq 1$ and $V$ has $m_{1}$ branches of type $B_{h_{1}}$ and $m-m_{1}$ branches of type $B_{h_{2}}$, where $h_{1}=\left\lfloor\frac{n-1-m}{2 m}\right\rfloor, h_{2}=\left\lceil\frac{n-1-m}{2 m}\right\rceil$ and $m_{1}=\frac{\left(3+2 h_{1}\right) m-(n-1)}{2}$ Figure (2). Here $\lfloor t\rfloor$ denotes the greatest integer less than $t$ and $\lceil t\rceil$ denotes the lowest integer greater than $t$.

Note that if a specific topological index is monotone with respect to the variation of gaps between branches, one extremal value of this index over $\tilde{\mathbf{K}} \mathbf{g}_{n, m}$ is attained in $V$ and the other extremal value is attained in $U$.

One of the indices which is monotone with respect to the variation of gaps in $\tilde{\mathbf{K}} \mathbf{g}_{n, m}$ is the energy. Given a graph $G$, its characteristic polynomial, denoted by $\Phi_{G}$, is defined as the characteristic polynomial of the adjacency matrix $A$ of $G$, i.e., $\Phi_{G}=\operatorname{det}(x I-A)$, where $I$ is the $n \times n$ identity matrix. The eigenvalues of $G$ are the eigenvalues of $A$. Note that for a graph $G$, its 
adjacency matrix $A$ is a real symmetric matrix and therefore the eigenvalues of $G$ are real numbers $\lambda_{1}, \ldots, \lambda_{n}$. The energy of $G$ is denoted by $E(G)$ and defined as $E(G)=\sum_{i=1}^{n}\left|\lambda_{i}\right|$. This concept was introduced in [9] and it is related to the total $\pi$-electron energy in a molecule represented by a (molecular) graph. For the chemical applications and the mathematical properties of the energy of a graph we refer, respectively, to the review [10] and the recent book [16].

In Theorem 2.1 we find that the maximal value of the energy over $\tilde{\mathbf{K}} \mathbf{g}_{n, m}$ is attained in the tree $V$ while the minimal value of the energy is attained in $U$.

The Wiener index also appears to be monotone with respect to the variation of gaps in $\tilde{\mathbf{K}} \mathbf{g}_{n, m}$. Let $u$ and $v$ be two vertices of the connected graph $G$. The distance between $u$ and $v$ is the length of a shortest path connecting $u$ and $v$. The Wiener index of the graph $G$, denoted by $W(G)$, is the sum of distances between all pairs of vertices of $G$.

The Wiener index of trees has been extensively studied (see review [2]). In the case of $\tilde{\mathbf{K}} \mathbf{g}_{n, m}$ we find that the maximal value of the Wiener index $\tilde{\mathbf{K}} \mathbf{g}_{n, m}$ is attained in the tree $V$ while the minimal value of the Wiener index is attained in $U$ (see Theorem 3.1).

In the last section of this work, we find the extremal values of several

vertex-degree-based topological indices over the set $\tilde{\mathbf{K}} \mathbf{g}_{n, m}$. We consider indices of the form $([5],[8],[14])$ :

$$
T I=T I(G)=\sum_{1 \leq i \leq j \leq n-1} m_{i j} \Psi(i, j)
$$

where $m_{i j}$ is the number of edges of the graph $G$ connecting a vertex of degree $i$ with a vertex of degree $j$. For instance, the following special cases of the function $\Psi(x, y)$ have been extensively used: $\Psi(x, y)=\frac{1}{\sqrt{x y}}$, for the Randić index [17]; $\Psi(x, y)=\sqrt{\frac{x+y-2}{x y}}$, for the atom-bond connectivity $(\mathrm{ABC})$ index $[3] ; \Psi(x, y)=x+y$, for the first Zagreb index [13] $\Psi(x, y)=$ $x y$, for the second Zagreb index [13]; $\Psi(x, y)=\frac{2 \sqrt{x y}}{x+y}$, for the geometricarithmetic index [19]; $\Psi(x, y)=\frac{1}{\sqrt{x+y}}$, for the sum-connectivity index [21] ; $\Psi(x, y)=\frac{(x y)^{3}}{(x+y-2)^{3}}$, for the augmented Zagreb index [4] ; and $\Psi(x, y)=$ $\frac{2}{x+y}$, for the harmonic index [22]. 
In Theorem 4.1 we find that the maximal value of the Randic index, the geometric-arithmetic index, the sum-connectivity index and the harmonic index over $\tilde{\mathbf{K}} \mathbf{g}_{n, m}$ is attained in $V$ while the minimal value of these indices is attained in $U$. Conversely, the maximal value of the atom-bondconnectivity index, the first Zagreb index and the second Zagreb index is attained in $U$ and the minimal value is attained in $V$.

\section{Extremal values of the energy over $\tilde{\mathbf{K}} \mathbf{g}_{n, m}$}

In order to determine the maximal and minimal value of the energy over $\tilde{\mathbf{K}} \mathbf{g}_{n, m}$, we use the method to compare the energy of bipartite graphs consisting in defining a quasi-order relation which depends on the expression for the characteristic polynomial (see [7]). More precisely, $G$ is a bipartite graph if and only if

$$
\Phi_{G}=x^{n}+\sum_{k=1}^{\left\lfloor\frac{n}{2}\right\rfloor}(-1)^{k} c_{2 k}(G) x^{n-2 k}
$$

where $c_{2 k}(G)$ is a nonnegative integer for every $k=1, \ldots,\left\lfloor\frac{n}{2}\right\rfloor$. Then we can define the relation

$$
G_{1} \preceq G_{2} \text { if and only if } c_{2 k}\left(G_{1}\right) \leq c_{2 k}\left(G_{2}\right)
$$

for every $k=1, \ldots,\left\lfloor\frac{n}{2}\right\rfloor$. If, in addition, there exists a $k$ such that $c_{2 k}\left(G_{1}\right)<$ $c_{2 k}\left(G_{2}\right)$ then we write $G_{1} \prec G_{2}$. Now, based on Coulson's integral formula [1], it can be shown [7] that for a bipartite graph $G$, the energy is increasing with respect to the quasi-order relation. In other words, if $G_{1} \prec G_{2}$ then $E\left(G_{1}\right)<E\left(G_{2}\right)$.

Theorem 2.1. The maximal (resp. minimal) value of the energy over $\tilde{\mathbf{K}} \mathbf{g}_{n, m}$ is attained in the tree $V$ (resp. $U$ ).

Proof. Let $X \in \tilde{\mathbf{K}} \mathbf{g}_{n, m}$ with branches $B_{k}, B_{l}$ where $k>l$. Let $Y \in$ $\tilde{\mathbf{K}} \mathbf{g}_{n, m}$ obtained from $X$ replacing the branch $B_{k}$ by $B_{k-1}$ and the branch $B_{l}$ by $B_{l+1}$ (see Figure 3 ). 

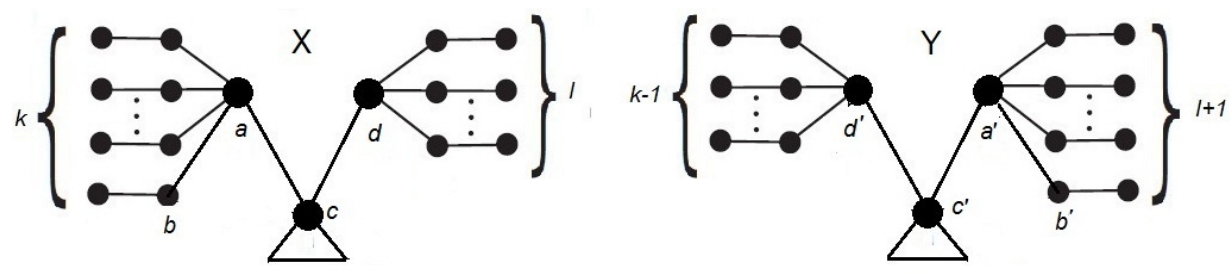

Figure 3 : Kragujevac trees used in the proof of Theorem 2.1

Denote by $\Phi_{X}$ the characteristic polynomial of the tree $X$. Removing the edge $a b$ using Schwenk's theorem [18] we have $\Phi_{X}=\Phi_{X-a b}-\Phi_{X-a-b}=$ $\left(x^{2}-1\right) \Phi_{Z}-x\left(x^{2}-1\right)^{k-1} \Phi_{W}$,

where $Z$ is the tree obtained from $X$ replacing the branch $B_{k}$ by $B_{k-1}$ and $W$ is the tree obtained from $X$ removing branch $B_{k}$. Now, removing the edge $c d$ from $W$ we obtain

$$
\Phi_{X}=\left(x^{2}-1\right) \Phi_{Z}-x\left(x^{2}-1\right)^{k-1}\left[\Phi_{T} \Phi_{B_{l}}-\left(x^{2}-1\right)^{l} \Phi_{T^{\prime}}\right],
$$

where $T$ is the tree obtained from $X$ removing the branches $B_{k}, B_{l}$ and $T^{\prime}$ is obtained from $T$ removing the central vertex $c$.

If we apply the same procedure to the tree $Y$, removing first the edge $a^{\prime} b^{\prime}$ we have $\Phi_{Y}=\left(x^{2}-1\right) \Phi_{Z}-x\left(x^{2}-1\right)^{l} \Phi_{W^{\prime}}$, where $W^{\prime}$ is the tree obtained from $Y$ removing branch $B_{l}$. Now, removing the edge $c^{\prime} d^{\prime}$ from $W^{\prime}$ we obtain the following expression for the characteristic polynomial of $Y$ :

$$
\Phi_{Y}=\left(x^{2}-1\right) \Phi_{Z}-x\left(x^{2}-1\right)^{l}\left[\Phi_{T} \Phi_{B_{k-1}}-\left(x^{2}-1\right)^{k-1} \Phi_{T^{\prime}}\right] .
$$

It is easy to see that the characteristic polynomial of a branch $B_{s}$ is $\Phi_{B_{s}}=$ $x\left(x^{2}-1\right)^{s-1}\left(x^{2}-s-1\right)$; then replacing the characteristic polynomial of $B_{l}$ in (2.3), the characteristic polynomial of $B_{k-1}$ in (2.4) and taking the difference $\Phi_{X}-\Phi_{Y}$ we obtain $\Phi_{X}-\Phi_{Y}=(l-k+1) x^{2}\left(x^{2}-1\right)^{k+l-2} \Phi_{T}$.

Since $T$ is a tree, i.e. bipartite graph, the polynomial $\Phi_{T}$ is of the form bip. It implies that the polynomial in the previous equation is also of the form bip and its first coefficient is negative because $l<k-1$. Then, using the quasiorder relation qo we have that $X \prec Y$, and $E(X)<E(Y)$. 
It means that when we have a tree in $\tilde{\mathbf{K}} \mathbf{g}_{n, m}$ with two branches with gap greater that one, we can construct another tree in the same class with greater value of the energy, by reducing the gap between branches, and with less value of the energy, by increasing the gap between branches. Then, the tree with maximal energy is $V$ while the tree with minimal energy is $U$.

\section{Extremal values of the Wiener index over $\tilde{\mathbf{K}} \mathbf{g}_{n, m}$}

Theorem 3.1. The maximal (respectively minimal) value of the Wiener index over $\tilde{\mathbf{K}} \mathbf{g}_{n, m}$ is attained in the tree $V$ (respectively $U$ ).

Proof. Let $X$ and $Y$ be the trees in $\tilde{\mathbf{K}} \mathbf{g}_{n, m}$ depicted in Figure 3. To find the difference $W(X)-W(Y)$ we use the following expression for a Wiener index of a tree $[20]$ :

$\mathrm{W}(\mathrm{T})=\sum_{e} n_{1}(e) n_{2}(e)$, where $T$ is a tree, summation goes for all edges of $T$ and $n_{1}(e)$ and $n_{2}(e)$ are the numbers of vertices lying on the two sides of the edge $e$.

Hence, in the difference $W(X)-W(Y)$ we have only to consider the edges $a c$ and $c d$ of $X$ and the edges $d^{\prime} c^{\prime}$ and $c^{\prime} a^{\prime}$ of $Y$ (see Figure 3):

$$
\begin{aligned}
W(X)-W(Y)= & (2 k+1)(n-2 k-1)+(2 l+1)(n-2 l-1) \\
& -(2 k-1)(n-2 k+1)-(2 l+3)(n-2 l-3) \\
= & 8 l-8 k+8=8(l-k+1)<0 .
\end{aligned}
$$

It means that when we have a tree in $\tilde{\mathbf{K}} \mathbf{g}_{n, m}$ with two branches with gap greater that one, we can construct another tree in the same class with greater value of the Wiener index, by reducing the gap between branches, and with less value of the Wiener index, by increasing the gap between branches. Then, the tree with maximal Wiener index is $V$ while the tree with minimal Wiener index is $U$.

\section{Extremal values of some vertex-degree-based topological indices over $\tilde{\mathbf{K}} \mathbf{g}_{n, m}$}

Theorem 4.1. The maximal (respectively minimal) value of the Randic index, the geometric-arithmetic index, the sum-connectivity index and the harmonic index over $\tilde{\mathbf{K}} \mathbf{g}_{n, m}$ is attained in the tree $V$ (respectively $U$ ). 
The maximal (respectively minimal) value of the atom-bond-connectivity index, the first Zagreb index and the second Zagreb index over $\tilde{\mathbf{K}} \mathbf{g}_{n, m}$ is attained in the tree $U$ (respectively $V$ ).

Proof. Let $X$ and $Y$ be the trees in $\tilde{\mathbf{K}} \mathbf{g}_{n, m}$ depicted in Figure 3. Using the expresion gen for the general vertex-degree-based topological index we have:

$$
\begin{aligned}
\Delta= & T I(X)-T I(Y) \\
= & {[\Psi(k+1, m)+\Psi(l+1, m)+k \Psi(k+1,2)+l \Psi(l+1,2)] } \\
& -[\Psi(k, m)+\Psi(l+2, m)+(l+1) \Psi(l+2,2)+(k-1) \Psi(k, 2)] \\
= & {[\Psi(k+1, m)-\Psi(k, m)+k \Psi(k+1,2)-(k-1) \Psi(k, 2)] } \\
& -[\Psi(l+2, m)-\Psi(l+1, m)+(l+1) \Psi(l+2,2)-l \Psi(l+1,2)] \\
= & F(k)-F(l+1)
\end{aligned}
$$

where $\mathrm{F}(\mathrm{x})=\Psi(x+1, m)-\Psi(x, m)+x \Psi(x+1,2)-(x-1) \Psi(x, 2)$.For $k=l+1, T I(X)-T I(Y)=0$. Assume that $k>l+1$. Note that for each of the indices considered in this theorem, for a fixed $y$ the function $\Psi$ is continuous and differentiable as a function of $x$, for $x>0$. It means that the function $F(x)$ is continuous in $[l+1, k]$ and differentiable in $(l+1, k)$. By the mean value theorem there exists $\xi \in(l+1, k)$ such that

$$
\begin{aligned}
\Delta= & F(k)-F(l+1)=F^{\prime}(\xi)(k-l-1) \\
= & {\left[\frac{\partial \Psi}{\partial x}(\xi+1, m)+\Psi(\xi+1,2)+\xi \frac{\partial \Psi}{\partial x}(\xi+1,2)\right](k-l-1) } \\
& -\left[\frac{\partial \Psi}{\partial x}(\xi, m)+\Psi(\xi, 2)+(\xi-1) \frac{\partial \Psi}{\partial x}(\xi, 2)\right](k-l-1) \\
= & {[G(\xi+1)-G(\xi)](k-l-1) }
\end{aligned}
$$

where

$\mathrm{G}(\mathrm{x})=\frac{\partial \Psi}{\partial x(x, m)+\Psi(x, 2)+(x-1) \frac{\partial \Psi}{\partial x}(x, 2) .}$ Now, since for all the considered indices, $\frac{\partial \Psi}{\partial x}$ is also continuous and differentiable as a function of $x$, for $x>0$, the function $G$ is continuous in the closed interval $[\xi, \xi+1]$ and differentiable in the open interval $(\xi, \xi+1)$. By the mean value theorem there exists $z \in(\xi, \xi+1)$ such that

$$
\begin{aligned}
\Delta & =G^{\prime}(z)(k-l-1) \\
& =\left[\frac{\partial^{2} \Psi}{\partial x^{2}}(z, m)+2 \frac{\partial \Psi}{\partial x}(z, 2)+(z-1) \frac{\partial^{2} \Psi}{\partial x^{2}}(z, 2)\right](k-l-1) .
\end{aligned}
$$


If for a specific vertex-degree-based topological index,

$$
T(x)=\frac{\partial^{2} \Psi}{\partial x^{2}}(x, m)+2 \frac{\partial \Psi}{\partial x}(x, 2)+(x-1) \frac{\partial^{2} \Psi}{\partial x^{2}}(x, 2) \leq 0
$$

for all $x \geq 1$, then $\Delta=T I(X)-T I(Y) \leq 0$ for every value of $k>l$ It means that the tree with maximal index is $V$ while the tree with minimal index is $U$. Conversely, if for a specific vertex-degree-based topological in$\operatorname{dex} T(x) \geq 0$ for all $x \geq 1$, then the tree with maximal index is $U$ while the tree with minimal index is $V$. In the next table we show that for Randić index, the geometric-arithmetic index, the sum-connectivity index and the harmonic index, $T(x) \leq 0$, while for the atom-bond-connectivity index, the first Zagreb index and the second Zagreb index, $T(x) \geq 0$.

\begin{tabular}{|l|r|}
\hline Index & $T(x)$ \\
\hline Randić & $\frac{-x \sqrt{m}-3(\sqrt{m}-\sqrt{2})}{4 x^{2} \sqrt{2 m x}}<0$ \\
Geometric-arithmetic & $-\frac{\sqrt{m}\left(m^{2}+6 m x-3 x^{2}\right)}{2 x \sqrt{x}(m+x)^{3}}-\frac{\sqrt{2}\left(x^{3}+15 x^{2}-24 x-4\right)}{2 x \sqrt{x}(2+x)^{3}}<0$ \\
Sum-connectivity & $-\frac{\left((m+x)^{\frac{5}{2}}(11+x)-3(x+2)^{\frac{5}{2}}\right)}{4(x+2)^{\frac{5}{2}}(m+x)^{\frac{5}{2}}}<0$ \\
Harmonic & $-\frac{4\left[\left(3 m^{3}+2 x^{3}-8\right)+3 x^{2}(3 m-2)+3 x\left(3 m^{2}-4\right)\right]}{(x+2)^{3}(m+x)^{3}}<0$ \\
Atom-bond connectivity & $\frac{(m-2)(4 x+3 m-6)}{4 x^{2}(x+m-2) \sqrt{x m(x+m-2)}}>0$ \\
First Zagreb & $2>0$ \\
Second Zagreb & $4>0$ \\
\hline
\end{tabular}

\section{References}

[1] C. A. Coulson, On the calculation of the energy in unsaturated hydrocarbon molecules, Proc. Cambridge Phil. Soc., 36, pp. 201-203, (1940). 
[2] A. A. Dobrynin, R. Entriguer and I. Gutman, Wiener index of trees: Theory and applications, Acta Appl. Math, 66, pp. 211-249, (2001).

[3] E. Estrada, L. Torres, L. Rodríguez and I. Gutman, An atom-bond connectivity index: Modelling the enthalpy of formation of alkanes, Indian J. Chem., 37A, pp. 849-855, (1998).

[4] B. Furtula, A. Graovac and D. Vukičević, Augmented Zagreb index, J. Math. Chem., 48, pp. 370-380, (2010).

[5] B. Furtula, I. Gutman and M. Dehmer, On structure-sensitivity of degree-based topological indices, Appl. Math. Comput., 219, pp. 89738978, (2013).

[6] B. Furtula, I. Gutman, M. Ivanović and D. Vukićević, Computer search for trees with minimal ABC index, Appl. Math. Comput., 219, pp. 767-772, (2012).

[7] I. Gutman, Acyclic systems with extremal Hückel $\pi$-electron energy, Theor. Chim. Acta, 45, pp. 79-87, (1977).

[8] I. Gutman, Degree-based topological indices, Croat. Chem. Acta, 86, pp. 351-361, (2013).

[9] I. Gutman, The energy of a graph, Ber. Math.-Statist. Sekt. Forschungsz. Graz, 103, pp. 1-22, (1978).

[10] I. Gutman, Topology and stability of conjugated hydrocarbons. The dependence of total $\pi$-electron energy on molecular topology, J. Serb. Chem. Soc., 70, pp. 441-456, (2005).

[11] I. Gutman and B. Furtula, Trees with smallest atom-bond connectivity index, MATCH Commun. Math. Comput. Chem., 68, pp. 131-136, (2012).

[12] I. Gutman, B. Furtula and M. Ivanović, Notes on trees with minimal atom-bond connectivity index, MATCH Commun. Math. Comput. Chem., 67, pp. 467-482, (2012).

[13] I. Gutman and N. Trinajstić, Graph theory and molecular orbitals. Total $\pi$-electron energy of alternant hydrocarbons, Chem. Phys. Lett., 17, pp. 535-538, (1972). 
[14] B. Horoldagva and I. Gutman, On some vertex-degree-based graph invariants, MATCH Commun. Math. Comput. Chem., 65, pp. 723$730,(2011)$.

[15] S. A. Hosseini, M. B. Ahmadi and I. Gutman, Kragujevac Trees with minimal Atom-Bond Connectivity Index, MATCH Commun. Math. Comput. Chem., 71, pp. 5-20, (2014).

[16] X. Li, Y. Shi and I. Gutman, Graph Energy, Springer, New York, (2012).

[17] M. Randić, On characterization of molecular branching, J. Am. Chem. Soc., 97, pp. 6609-6615, (1975).

[18] A. Schwenk, Computing the characteristic polynomial of a graph, in: Lectures Notes in Mathematics, vol 406, Springer-Verlag, Berlin, Heidelberg, pp. 153-172, (1974).

[19] D. Vukičević and B. Furtula, Topological index based on the ratios of geometrical and arithmetical means of end-vertex degrees of edges, J. Math. Chem., 46, pp. 1369-1376, (2009).

[20] H. Wiener, Structural determination of paraffin boiling points, J. Amer. Chem. Soc., 69, pp. 17-20, (1947).

[21] B. Zhou and N. Trinajstić, On a novel connectivity index, J. Math. Chem., 46, pp. 1252-1270, (2009).

[22] L. Zhong, The harmonic index for graphs, Appl. Math. Lett., 25, pp. 561-566, (2012).

\section{Roberto Cruz}

Instituto de Matemáticas, Universidad de Antioquia

Medellín,

Colombia

e-mail : roberto.cruz@udea.edu.co

\section{Ivan Gutman}

Faculty of Science,

University of Kragujevac

Kragujevac,

Serbia

e-mail : gutman@kg.ac.rs

and 


\section{Juan Rada}

Instituto de Matemáticas,

Universidad de Antioquia

Medellín,

Colombia

e-mail : pablo.rada@udea.edu.co 